January 5, 2018

\title{
Analysis of haplotype networks: the randomized minimum spanning tree method
}

Emmanuel Paradis

ISEM, IRD, Univ. Montpellier, CNRS, EPHE, Montpellier, France

E-mail: Emmanuel.Paradis@ird.fr 


\section{Summary}

1. Haplotype network construction is a widely used approach for analysing and visualising the relationships among DNA sequences within a population or species. This approach has some problems such as how to quantify alternative links among sequences, or how to plot efficiently networks to compare them easily.

2. In this paper, a new method is presented: the randomized minimum spanning tree method, based on randomizing the input order of the data in order to produce alternative branchings in the haplotype network. It is shown that this new method can produce, at least in some situations, networks with less alternative links than the minimum spanning network method.

3. A new graphical display of haplotype networks is introduced here. This is based on calculating the coordinates of the haplotypes from a multidimensional scaling of the haplotype distance matrix. The display can be done in two or three dimensions. The eigenvalues extracted from the multidimensional scaling analysis give an indication of the relevant number of dimensions.

4. These tools are illustrated with the analyses of published data on the leopard and on the jaguar. These analyses show interesting and contrasting patterns between these two species of big cats.

5. All tools are implemented in $\mathrm{R}$ and available in the package pegas.

Keywords: Hamming distance, haplotype network, microevolution, minimum spanning 


\section{Introduction}

The analysis of DNA sequences from individuals sampled in one or several populations likelihood, Bayesian inference, split decomposition, or consensus methods (Holland 
et al., 2004). Another important criterion is whether unobserved haplotypes can be included in the network: distance-based methods cannot generally do this because they do not consider explicitly the evolving characters (Table 1). It is crucial to assess the reliability of a phylogeny or network of haplotypes since its construction may be affected by sampling biases (i.e., missing haplotypes). A badly estimated network is likely to lead to wrong inference of population or history.

The present paper focuses on the distance-based, implicit network approach. Two methods are commonly used to build a haplotype network: the minimum spanning tree (MST) method (Kruskal, 1956) and the method from Templeton et al. (1992, TCS). The MST method has been applied in many fields (Nešetřil et al., 2001). Its principle is to first build a matrix of pairwise distances among sequences (or haplotypes), and then find the shortest set of paths that link all observations where the length of each link is taken from the pairwise distance. The TCS method, often referred to as statistical parsimony, is based on a model of evolution of the genetic characters measured on each individual—originally restriction fragment lengths but the method can be applied to DNA sequences. The main difference between both methods is that an MST is a network with no reticulation, thus for $n$ sequences, the resulting MST will have $n-1$ links. On the other hand, a TCS network may have reticulations defining alternative branchings, and include unobserved haplotypes in the network. Thus, this method may be used to infer micro-evolutionary events such as putative recombinations (Posada \& Crandall, 2001). Bandelt et al. (1999) developed another method called the median-joining network (MJN) where alternative branchings are found by examining potential ancestral sequences for each triplet of sequences. MJN belongs to a class of methods which includes several variants such as reduced median network or quasi-median network (Bandelt et al., 1995, 1999). Thus, the MST usually cannot represent possible ambiguous or alternative branchings. Another limitation of the MST construction is that, 
for a given data set, the MST may not be unique. Figure 1 shows a simple illustration of

\section{Methods}

\section{MINIMUM SPANNING TREE AND NETWORK}

The MST algorithm can be sketched as follows (Kruskal, 1956):

1. Compute the matrix of pairwise distances among the $n$ observations and sort them in increasing order.

2. Assign each observation to its own group; there are thus $n$ initial groups.

$$
\text { 3. Set } i \leftarrow 1 \text {. }
$$

4. Take the $i$ th distance from step 1: if the two corresponding observations are not in the same group, then create a link between them and pool the two groups.

\section{Set $i \leftarrow i+1$.}

6. Repeat steps 4 and 5 until there is a one group. 
The number of groups is decreased by one at each iteration of step 4 . The issue with this

of length one A-C and A-B; the second step considers the links of length two A-D and 
B-C. During the MST construction, the link B-C is never included because B and C were already grouped together during the first step, so the RMST does not include this link. On the other hand, the MSN includes this link thus resulting in two alternative paths, B-A-C and B-C, both of the same length. The RMST avoids this ambiguity. Note the difference between the present example and the one in Figure 1: in the latter, the additional link output by the MSN creates a path shorter than the one created by the MST.

\section{GRAPHICAL TOOLS}

Plotting networks is a notoriously difficult problem (e.g., Kloepper \& Huson, 2008). Several computer programs perform graphical display of various types of evolutionary networks, such as igraph (Csardi \& Nepusz, 2006), network (Butts, 2008), phangorn (Schliep, 2011), pegas (Paradis, 2010), or SplitsTree (Huson, 1998), among many others. In practice, it would be very useful to graphically compare networks constructed under different methods or assumptions. However, this is usually not possible (or very difficult) because there is no standard procedure for plotting haplotype networks. To propose a solution to this problem, an implementation based on multidimensional scaling (MDS;

Torgerson, 1952) is developed here. The procedure is to first perform an MDS on the distance matrix in order to extract two or three sets of coordinates. These coordinates are then used to plot the observed sequences or haplotypes in 2-D or in 3-D, and the links inferred from the network are then drawn. Thus, this procedure contrasts with most existing ones which compute the layout of haplotypes trying to minimize line crossings (e.g., Kloepper \& Huson, 2008). The proposed procedure has several advantages. First, an MDS on the original distance matrix will arrange the sequences depending on their similarity. Second, the eigenvalues extracted from the MDS make possible to assess whether it is relevant to use two or three dimensions in this projection. Third, the 
coordinates of the observations will be the same for all networks since they depend only on the distance matrix, making graphical comparisons easier. Fourth, the procedure is computationally straightforward since an MDS is usually fast to perform even with several hundreds observations.

The tools presented in this article have been implemented in the $\mathrm{R}$ package pegas (Paradis, 2010). This package has already implemented a standard 2-D plot using an "energy-minimisation" algorithm to optimise the layout. Plots in three dimensions have been implemented using the rgl package (Adler et al., 2016).

\section{SIMULATION STUDY}

To assess how the RMST is able to find alternative links in a network of haplotypes, some simulations were run under different situations of mutation rate $(\mu)$, sequence length $(l)$, and number of sequences $(n)$. A set of $n$ sequences was simulated under the JC69 model of sequence evolution (Jukes \& Cantor, 1969) along a random network with no reticulation and link lengths taken from a standard uniform distribution. The network was simulated by generating a random binary tree where the internal nodes were considered contemporaneous to the leaves. The sequences were then analysed with the

RMST using different numbers of randomizations $(5,10,20,50,100)$ : the number of additional links found by the RMST as well as the number of unique distances were recorded. The other parameters were: $n=50,100,500$, or $1000, l=500$ or 1000 , and $\mu=0.01$ or 0.1 . These parameter values were chosen to result in substantial numbers of ties in the distance matrices. The simulations were replicated 100 times for each combination of $n, l$, and $\mu$. The code used is provided in the Supplementary Information. 


\section{DATA} Information.

\section{Results}

\section{SIMULATION STUDY}

Two data sets were considered to apply the methods introduced in this paper: a set of mtDNA sequences from 33 leopards (Panthera pardus) published by Uphyrkina et al. (2001), and a set of mtDNA sequences from 37 jaguars (P. onca) published by Eizirik et al. (2001). Both data sets were downloaded from GenBank (accession numbers: AY035227-AY035292 and AF244814-AF244887, for each species, respectively). The sequences were aligned separately for the different genes (using information from GenBank and from the original publications) with MUSCLE (Edgar, 2004), and then combined into two global alignments with 726 and 707 sites, respectively. All sequences were unique for the leopard data, but 22 unique sequences were identified for the jaguar data. For each alignment, a matrix of Hamming distances was calculated with ape (Paradis et al., 2004). These matrices were used as input for the construction of the networks. The individual labels from the original studies were kept for the present analyses. The R scripts used for these analyses is provided in the Supplementary as successive differences with increasing number of randomizations (i.e., the numbers of links found with five randomizations compared to zero, with ten randomizations compared to five, and so on). The results were clearly related to the number of unique distances among the simulated distances. Considering that the total number of distances is given by $n(n-1) / 2$, the percentage of unique distances was always less than $1 \%$ (Table 2). Increasing $l$ and/or $\mu$ resulted in more variation among the sequences and, 
consequently, less additional links for the same $n$. On the other hand, increasing $n$ for the same values of $l$ and $\mu$ resulted in more closely related sequences, and thus more

additional links found by the RMST. In three cases, some additional links were still

found with 100 randomizations. In the scenario simulating the most diversity $(l=1000$, $\mu=0.1$ ), five randomizations were enough to find the additional links of the RMST for all values of $n$.

\section{APPLICATION}

The MDS analysis of the distance matrices resulted in slightly different patterns of eigenvalues. For the leopard data, the first eigenvalue was much larger than the others though the second and third ones were substantially larger than the remaining ones (Fig. 3a). For the jaguar data, the first and the second eigenvalues were much larger than all the other ones (Fig. 3b). Thus, we may anticipate that three dimensions may represent the distribution of sequences for the leopard data whereas two dimensions may be enough for the jaguar data.

The MSN and RMST analyses revealed large numbers of additional links compared to the MST ones (Table 3). In the case of the leopards, the number of links was multiplied by 6.7 from the MST to the MSN, and by 1.9 to the RMST. This increase in number of links was slightly smaller in the case of the jaguar: 5 to the MSN and 1.3 to the RMST. The RMST analyses were repeated with different numbers of randomizations. For the leopard data, 59 links were found with 10 randomizations while 60 links were found with 50 randomizations or more. For the jaguar data, 28 links were found with 10 randomizations or more.

The MDS-based plots of the leopard sequences showed a clear continental separation with the African individuals (SHO*) on the right-hand side of the plot, and the Asian ones on the left-hand side (Fig. 4). Interestingly, two individuals laid outside of these 
two groups: the one from the Arabian Peninsula (NIM1) and the one from Java, Indonesia (MEL1). Remarkably, the RMST did not add any further link from NIM1 to the others, whereas two additional links were observed between MEL1 and the Asian ones. The MSN kept NIM1 with a single link to SAX2, but added one further link between MEL1 and the others. The 3-D displays revealed that the African individuals, which are apparently aligned in the 2-D plots, are actually arranged along an arc in the third dimension of the MDS (videos provided in the Supplementary Information).

For the jaguar data, the arrangement of the individuals on the first axis followed a North-South axis with individuals from the South on the right-hand side of the plot (Fig. 5). Two individuals remained single-linked with the MSN and RMST analyses: Pon23 from Nicaragua which was linked with two individuals from Nicaragua and from Costa Rica, both with the same haplotype, and Pon63 from Venezuela which was linked with Pon73, an individual of unknown origin but presumably from Brazil (Eizirik et al., 2001). Except for Pon63, very little dispersion was observed in the third dimension as expected from the eigenvalues of the MDS (videos provided in the Supplementary Information).

\section{Discussion}

The analysis of the relationships among DNA sequences and haplotypes within and among populations is crucial for testing hypotheses on microevolutionary processes. However, such analyses often suffer from shortcomings. Typically, two issues are often observed. First, practitioners usually construct a single haplotype network which is then interpreted depending on the context of the study. This can be a problem when the assumptions of the method are not met, which can usually be assessed by comparing different constructions, for instance, by using different distances. The second problem is that there seems to be a confusion in the literature between MST and MSN. It is common 
to read "minimum spanning network" when an MST is obviously shown since no loop is

present. This is problematic since the MST may not be unique as already mentioned by Bandelt et al. (1999).

The RMST method is an alternative to the MSN with the advantage of creating less links among haplotypes while fully taking into account the ambiguities induced by data ordering. In the applications with real data presented in this paper, 100 randomizations were done and the results were identical than with smaller numbers. The computing times of the method is thus proportional to the product of $n$ (since $n-1$ links are built at each replication of the MST algorithm) with the number of randomizations. The implementation in pegas resamples the distance matrix by reordering its rows and columns simultaneously, instead of reordering the original data matrix, and thus avoids to recalculate the distance matrix at each iteration of the MST (which requires a computing time proportional to $n^{2}$ ).

The simulation study showed that the number of randomizations required to reach convergence of the RMST procedure is affected by the number of sequences, the sequence length, and the mutation rate. Because it is not easy to define a priori how many randomizations are required for a given data set, it is recommended to repeat the analyses with increasing numbers of randomizations and check that the constructed networks are identical (see code in Supplementary Information).

The respective merits of the methods used to construct haplotype networks are still debated (e.g., Mardulyn, 2012). However, comparing different methods is not without difficulties because some of them construct networks that have inherently different structures. Parsimony-based methods seek to combine all most parsimonious phylogenetic trees into a network which, as a consequence of this estimation procedure, have the observed sequences only at its terminal nodes (Branders \& Mardulyn, 2016). This contrasts with, on one hand, the MST-based methods where the observed sequences 
are at both the internal and the terminal nodes of the network, and, on the other hand, the TCS or MJN method which includes unobserved sequences in the network. For instance, the MJN constructed with the data in Figure 1 would have four nodes and three links all of length one, but this network is not strictly different from the MSN and RMST ones (Fig. 1c) because they all define paths of length two between each pair of observed sequences. Clearly, the presence of loops in RMST or MSN networks must be interpreted cautiously with respect to potentially unsampled haplotypes (e.g., Joly et al., 2007). On the other hand, the MJN inferred from the data in Figure 2 would be identical to the RMST (Fig. 2d) but different from the MSN (Fig. 2c).

Plotting networks (i.e., graphs with reticulations) is notoriously difficult for graphical software developers. This is another difficulty in the analysis of haplotype relationships. The graphical approach proposed here is a solution to this problem. By using the coordinates inferred from the MDS applied on the distance matrix, the haplotypes are always positioned in the same way, for a given distance matrix, whatever the links among them. Furthermore, the analysis of the eigenvalues extracted from the MDS gives information on the general structure of the data as illustrated by the examples above.

The analyses of the leopard and the jaguar data were mainly illustrative, although they show some interesting results. For the leopards, the contrast between African and Asian individuals was very clear. Two individuals were outside the bulk of the other individuals: one from the Arabian Peninsula, and the other from Java. Both represent two subspecies (P. pardus nimr and P. pardus melas) that are morphologically markedly different from the others (Stein \& Hayssen, 2013). Variation within the African group was also substantial and appeared in the third dimension of the plot. For the jaguars, variation was much less than for the leopards, and the MSN and the RMST showed much more additional links than the MST. Interestingly, Eizirik et al. (2001) reported an MSN with only one additional link. However, the present analysis showed that the 
RMST had seven additional links and the MSN even more.

Another interesting result is the presence of a "horseshoe effect" with the leopard data but not with the jaguar data. This effect, which is sometimes observed in multivariate analyses like MDS or principal component analysis (PCA), is a consequence of the dominance of local structures in the data. Such dominance can be the result of the inherent structure of the original data matrix (Ahmed et al., 1974), a transformation of the distances that gives more emphasis on the most similar observations (e.g., an exponential decay function; Diaconis et al., 2008), or, typically for population genetic data, local processes such as isolation by distance (Novembre \& Stephens, 2008). Whatever the origin of such structures, the decomposition of the data matrix (in the case of PCA) or of the distance matrix (in the case of MDS) results in the second axis to be related to the first one in a polynomial-like manner (actually a sinusoidal function; see Ahmed et al., 1974; Diaconis et al., 2008), and the subsequent axes with increasing degrees of the polynomials. In practice, the proximities of the sequences on the second and third axes must therefore be interpreted with caution. However, this does not affect the interpretation of the network layouts which are the same for all networks as long as the distance matrix is the same.

As rightly pointed out by Leigh \& Bryant (2015), the haplotype network methodology does not generally rely on an evolutionary model. However, a distance-based approach is very valuable because distances can be computed for different kinds of data, and they are straightforward to interpret in terms of number of changes. An interesting perspective will be to develop an approach to incorporate models of DNA sequence evolution into haplotype network analyses. A challenge will be to find how to compute a likelihood in the presence of loops in the network (Maynard Smith, 1989). 


\section{Data accessibility}

The data are archived in GenBank (accession numbers: AY035227-AY035292 and AF244814-AF244887).

\section{References}

Adler, D., Murdoch, D., Nenadic, O., Urbanek, S., Chen, M., Gebhardt, A., Bolker, B., Csardi, G., Strzelecki, A., Senger, A. \& R Core Team (2016) rgl: 3D visualization using OpenGL. R package version 0.95.1441.

Ahmed, N., Natarajan, T. \& Rao, K.R. (1974) Discrete cosine transform. IEEE

I am grateful to the Associate Editor and three anonymous reviewers for their constructive comments on a previous version of this paper. The simulations benefited from the ISEM computing cluster platform. This is publication ISEM 2017-277.

\section{Transactions on Computers, C-23, 90-93.}

Bandelt, H.J., Forster, P. \& Röhl, A. (1999) Median-joining networks for inferring intraspecific phylogenies. Molecular Biology and Evolution, 16, 37-48.

Bandelt, H.J., Forster, P., Sykes, B.C. \& Richards, M.B. (1995) Mitochondrial portraits of human populations using median networks. Genetics, 141, 743-753.

Bossart, J.L. \& Prowell, D.P. (1998) Genetic estimates of population structure and gene flow: limitations, lessons and new directions. Trends in Ecology \& Evolution, 13, 202-206.

Branders, V. \& Mardulyn, P. (2016) Improving intraspecific allele networks inferred by maximum parsimony. Methods in Ecology \& Evolution, 7, 90-95.

\section{Acknowledgements}


Buerkle, C.A. \& Lexer, C. (2008) Admixture as the basis for genetic mapping. Trends in Ecology \& Evolution, 23, 686-694.

Butts, C. (2008) network: a package for managing relational data in R. Journal of Statistical Software, 24, 2.

Csardi, G. \& Nepusz, T. (2006) The igraph software package for complex network research. InterJournal, Complex Systems, 1695.

Diaconis, P., Goel, S. \& Holmes, S. (2008) Horseshoes in multidimensional scaling and local kernel methods. Annals of Applied Statistics, 2, 777-807.

Edgar, R.C. (2004) MUSCLE: multiple sequence alignment with high accuracy and high throughput. Nucleic Acids Research, 32, 1792-1797.

Eizirik, E., Kim, J.H., Menotti-Raymond, M., Crawshaw, Jr, P.G., O’Brien, S.J. \& Johnson, W.E. (2001) Phylogeography, population history and conservation genetics of jaguars (Panthera onca, Mammalia, Felidae). Molecular Ecology, 10, 65-79.

Emerson, B., Paradis, E. \& Thébaud, C. (2001) Revealing the demographic histories of species using DNA sequences. Trends in Ecology \& Evolution, 16, 707-716.

Holland, B.R., Huber, K.T., Moulton, V. \& Lockhart, P.J. (2004) Using consensus networks to visualize contradictory evidence for species phylogeny. Molecular Biology and Evolution, 21, 1459-1461.

Huson, D.H. (1998) SplitsTree: analyzing and visualizing evolutionary data. Bioinformatics, 14, 68-73.

Huson, D.H. \& Bryant, D. (2006) Application of phylogenetic networks in evolutionary studies. Molecular Biology and Evolution, 23, 254-267.

Joly, S., Stevens, M.I. \& van Vuuren, B.J. (2007) Haplotype networks can be misleading in the presence of missing data. Systematic Biology, 56, 857-862.

Jombart, T., Eggo, R.M., Dodd, P.J. \& Balloux, F. (2011) Reconstructing disease outbreaks from genetic data: a graph approach. Heredity, 106, 383-390. 
Jukes, T.H. \& Cantor, C.R. (1969) Evolution of protein molecules. H.N. Munro, ed., Mammalian Protein Metabolism, pp. 21-132. Academic Press, New York.

Kloepper, T.H. \& Huson, D.H. (2008) Drawing explicit phylogenetic networks and their integration into SplitsTree. BMC Evolutionary Biology, 8, 22.

Kruskal, Jr, J.B. (1956) On the shortest spanning subtree of a graph and the traveling salesman problem. Proceedings of the American Mathematical Society, 7, 48-50.

Leigh, J.W. \& Bryant, D. (2015) POPART: full-feature software for haplotype network construction. Methods in Ecology \& Evolution, 6, 1110-1116.

Mardulyn, P. (2012) Trees and/or networks to display intraspecific DNA sequence variation? Molecular Ecology, 21, 3385-3390.

Maynard Smith, J. (1989) Trees, bundles or nets? Trends in Ecology \& Evolution, 4, 302-304.

Nešetřil, J., Milková, E. \& Nevsetřilová, H. (2001) Otakar Borůvka on minimum spanning tree problem Translation of both the 1926 papers, comments, history. Discrete Mathematics, 233, 1-36.

Novembre, J. \& Stephens, M. (2008) Interpreting principal component analyses of spatial population genetic variation. Nature Genetics, 40, 646-649.

Paradis, E. (2010) pegas: an R package for population genetics with an

integrated-modular approach. Bioinformatics, 26, 419-420.

Paradis, E., Claude, J. \& Strimmer, K. (2004) APE: analyses of phylogenetics and evolution in R language. Bioinformatics, 20, 289-290.

Posada, D. \& Crandall, K.A. (2001) Intraspecific gene genealogies: trees grafting into networks. Trends in Ecology \& Evolution, 16, 37-45.

R Core Team (2017) R: A Language and Environment for Statistical Computing. R Foundation for Statistical Computing, Vienna, Austria.

Schliep, K.P. (2011) phangorn: phylogenetic analysis in R. Bioinformatics, 27, 592-593. 
Stein, A.B. \& Hayssen, V. (2013) Panthera pardus (Carnivora: Felidae). Mammalian

onca_RMST.mp4. Animation of the RMST from the jaguar data. 
Table 1: Comparison of some features of different methods to contruct haplotype networks. MST: minimum spanning tree; MSN: minimum spanning network; RMST: randomized minimum spanning tree; TCS: statistical parsimony; MP: maximum parsimony; MJN: median-joining network; $n$ : number of haplotypes; $L$ : number of links in the network.

\begin{tabular}{lccll}
\hline Method & Input data & $L$ & Unobserved haplotypes & Reference \\
\hline MST & Distances & $n-1$ & No & Kruskal (1956) \\
MSN & $\prime \prime$ & $\geq n-1$ & No & Bandelt et al. (1999) \\
RMST & $\prime \prime$ & $\prime \prime$ & No & This paper \\
TCS & Sequences & $\prime \prime$ & Possibly & Templeton et al. (1992) \\
MP & $\prime \prime$ & $\prime \prime$ & Yes, at internal nodes & Branders \& Mardulyn (2016) \\
MJN & $\prime \prime$ & $\prime \prime$ & Possibly, as median-vectors & Bandelt et al. (1999) \\
\hline
\end{tabular}

Table 2: Simulation results: mean number of additional links found by increasing the number of randomizations in the RMST algorithm ( $l$ : sequence length; $\mu$ : mutation rate; $n$ : number of sequences; NUD: mean number of unique distances).

\begin{tabular}{|c|c|c|c|c|c|c|c|c|}
\hline \multirow[t]{2}{*}{$l$} & \multirow{2}{*}{$\mu$} & \multirow[t]{2}{*}{$n$} & \multicolumn{5}{|c|}{ Number of randomizations } & \multirow[t]{2}{*}{ NUD } \\
\hline & & & 5 & 10 & 20 & 50 & 100 & \\
\hline \multirow{8}{*}{500} & \multirow{4}{*}{0.01} & 50 & 29.96 & 7.79 & 3.24 & 1.16 & 0.04 & 39.1 \\
\hline & & 100 & 60.25 & 15.62 & 6.78 & 2.75 & 0.31 & 0.40 \\
\hline & & 500 & 302.88 & 74.31 & 33.65 & 12.29 & 1.61 & 0.24 \\
\hline & & 1000 & 613.10 & 156.06 & 75.05 & 28.22 & 3.44 & 94.85 \\
\hline & \multirow[t]{4}{*}{0.1} & 50 & 2.48 & 0.18 & 0.02 & 0.00 & 0.00 & 216.09 \\
\hline & & 100 & 5.71 & 0.52 & 0.01 & 0.00 & 0.00 & 270.74 \\
\hline & & 500 & 33.26 & 2.89 & 0.25 & 0.04 & 0.00 & 340.92 \\
\hline & & 1000 & 63.49 & 4.96 & 0.38 & 0.04 & 0.00 & 363.05 \\
\hline \multirow[t]{8}{*}{1000} & \multirow[t]{4}{*}{0.01} & 50 & 14.58 & 1.78 & 0.68 & 0.07 & 0.01 & 71.25 \\
\hline & & 100 & 26.42 & 3.61 & 0.79 & 0.14 & 0.00 & 96.87 \\
\hline & & 500 & 147.65 & 23.23 & 7.15 & 1.58 & 0.00 & 149.60 \\
\hline & & 1000 & 292.88 & 45.67 & 11.77 & 2.46 & 0.16 & 177.73 \\
\hline & \multirow[t]{4}{*}{0.1} & 50 & 1.33 & 0.21 & 0.00 & 0.00 & 0.00 & 390.53 \\
\hline & & 100 & 2.7 & 0.23 & 0.00 & 0.00 & 0.00 & 520.35 \\
\hline & & 500 & & 1.58 & 0.05 & 0.00 & 0.00 & 671.83 \\
\hline & & 1000 & 31.22 & 2.18 & 0.11 & 0.00 & 0.00 & 707.06 \\
\hline
\end{tabular}


Table 3: Number of links in the networks constructed with the two data sets analysed. $n$ : number of haplotypes.

\begin{tabular}{lcccc}
\hline \multirow{2}{*}{ Species } & $n$ & \multicolumn{3}{c}{ Number of links } \\
\cline { 3 - 5 } & & MST & MSN & RMST \\
\hline Leopard & 33 & 32 & 214 & 60 \\
Jaguar & 22 & 21 & 105 & 28 \\
\hline
\end{tabular}


(a)
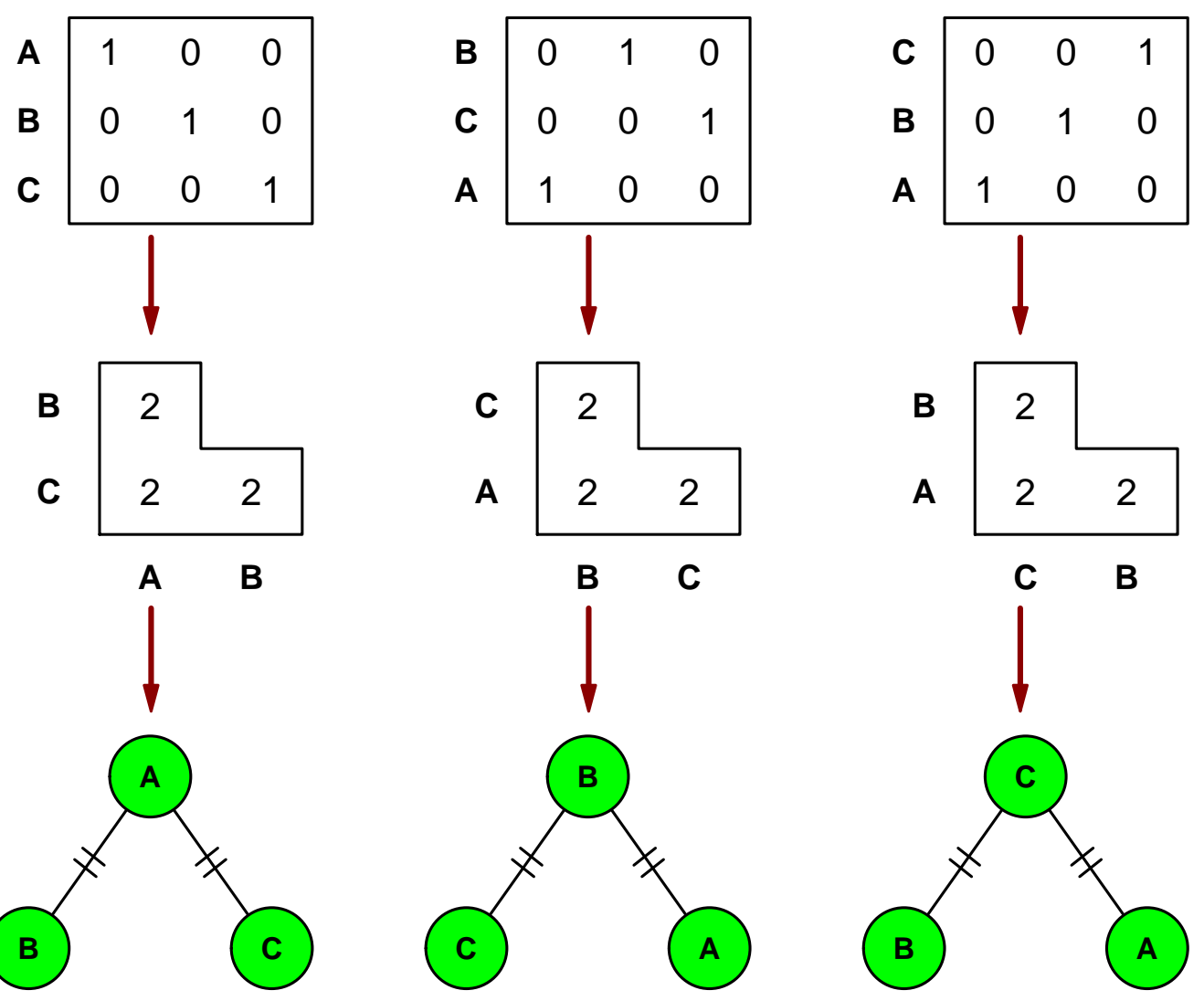

(b)

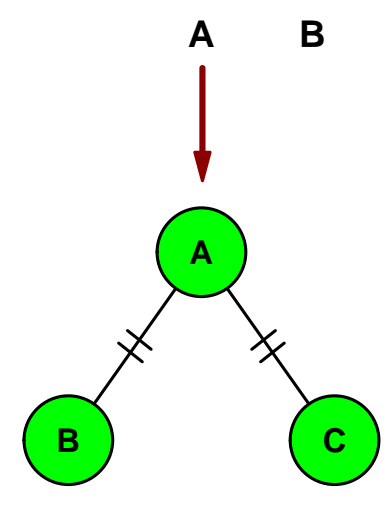

(c)

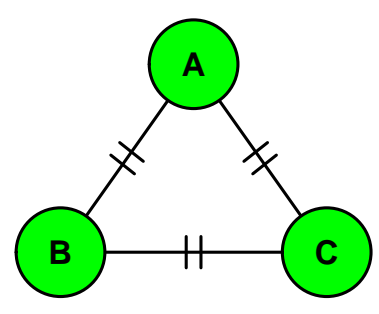

Figure 1: (a) Three identical data sets but with rows in different order and the corresponding distance matrices. (b) The three minimum spanning trees (MST) are different. (c) The minimum spanning network (MSN) and the randomized minimum spanning tree (RMST) are identical for the three data sets. 
(a)

\begin{tabular}{lllll|} 
A & 1 & 1 & 0 & 0 \\
B & 0 & 1 & 0 & 0 \\
C & 1 & 0 & 0 & 0 \\
D & 1 & 1 & 1 & 1 \\
\hline
\end{tabular}

(c)

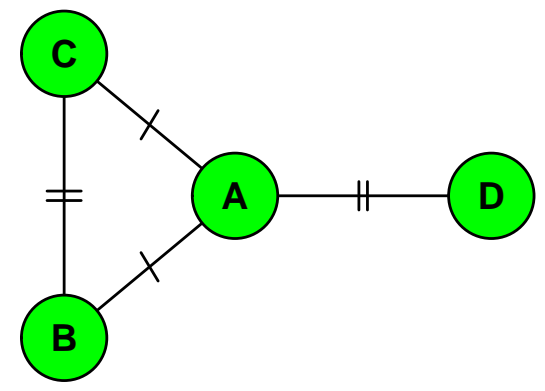

(b)

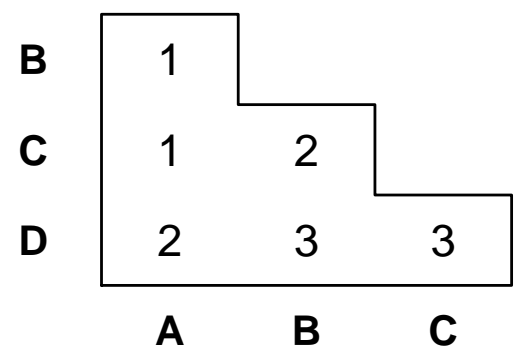

(d)

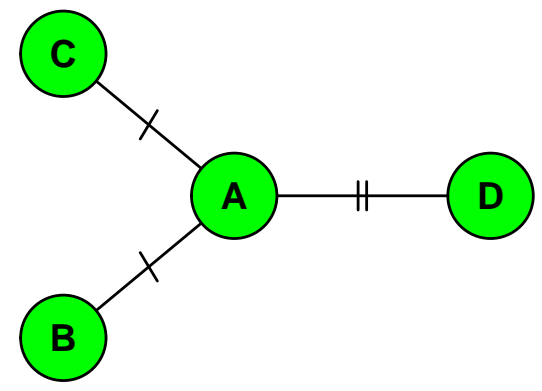

Figure 2: (a) A matrix of four sequences with four sites. (b) The inferred distances. (c) The minimum spanning network (MSN) creates a link between $\mathrm{B}$ and $\mathrm{C}$ when checking the distances of length two which has the same length than the path B-A-C. (d) The randomized minimum spanning tree (RMST) does not have additional link and is identical to the minimum spanning tree (MST). 
(a)

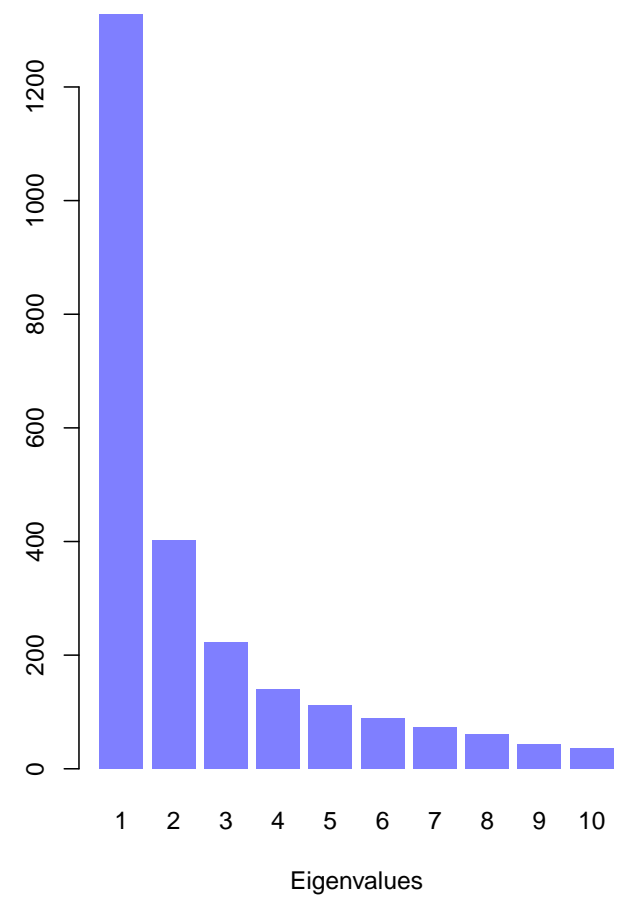

(b)

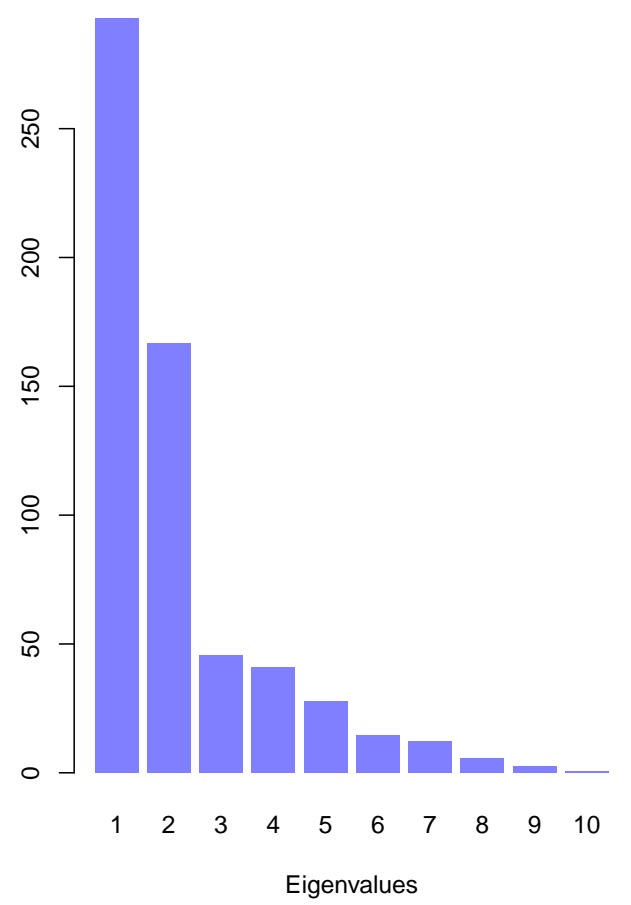

Figure 3: Eigenvalues extracted from the distance matrix for (a) leopards and (b) jaguars. 


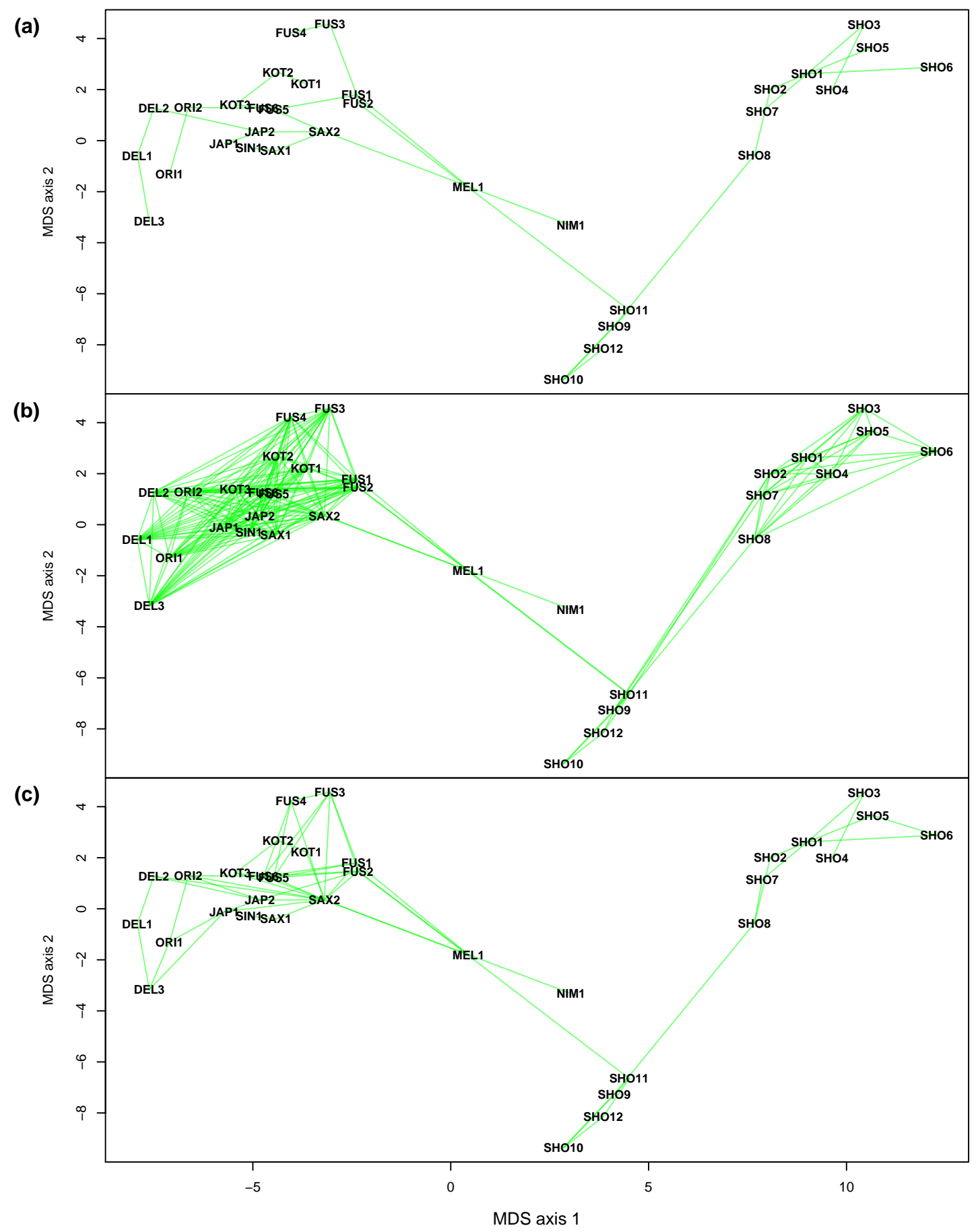

Figure 4: (a) Minimum spanning tree, (b) minimum spanning network, and (c) randomized minimum spanning tree for the leopard data. 


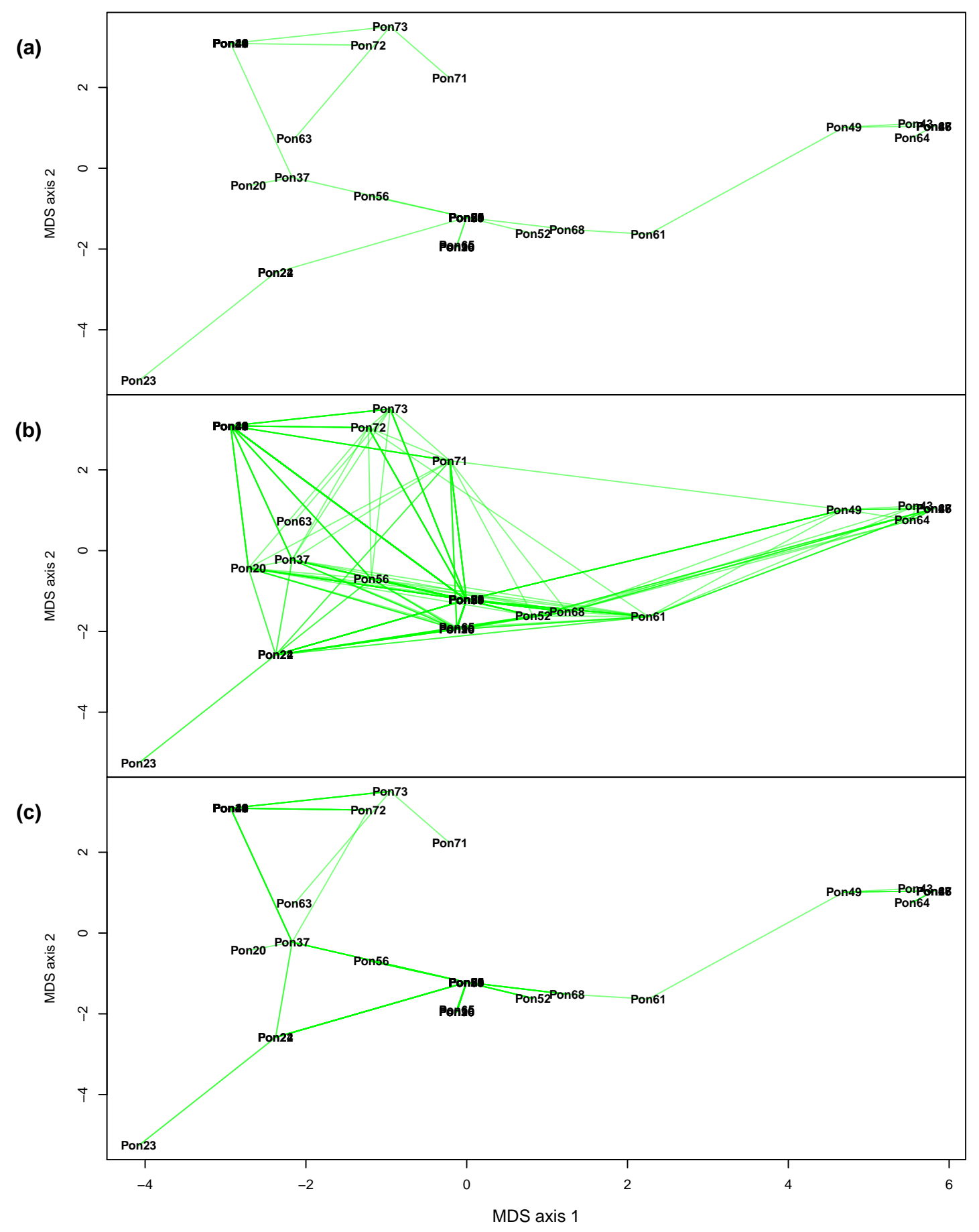

Figure 5: (a) Minimum spanning tree, (b) minimum spanning network, and (c) randomized minimum spanning tree for the jaguar data. 